\title{
Fiber-Optic Sensor for Aircraft Lightning Current Measurement
}

\author{
Truong X. Nguyen ${ }^{1}$, Jay J. Ely ${ }^{2}$ and George G. Szatkowski ${ }^{3}$ \\ NASA Langley Research Center, Hampton , VA 23681 \\ Carlos T. Mata ${ }^{4}$ and Angel G. Mata ${ }^{5}$ \\ ESC Kennedy Space Center, KSC, FL 32899 \\ and \\ Gary P. Snyder ${ }^{6}$ \\ NASA Kennedy Space Center, KSC, FL 32899
}

\begin{abstract}
An electric current sensor based on Faraday rotation effect in optical fiber was developed for measuring aircraft lightning current. Compared to traditional sensors, the design has many advantages including the ability to measure total current and to conform to structure geometries. The sensor is also small, light weight, non-conducting, safe from interference, and free of hysteresis and saturation. Potential applications include characterization of lightning current waveforms, parameters and paths, and providing environmental data for aircraft certifications. In an optical fiber as the sensing medium, light polarization rotates when exposed to a magnetic field in the direction of light propagation. By forming closed fiber loops around a conductor and applying Ampere's law, measuring the total light rotation yields the enclosed current. A reflective polarimetric scheme is used, where polarization change is measured after the polarized light travels round-trip through the sensing fiber. The sensor system was evaluated measuring rocket-triggered lightning over the 2011 summer. Early results compared very well against a reference current shunt resistor, demonstrating the sensor's accuracy and feasibility in a lightning environment. While later comparisons show gradually increasing amplitude deviations for an undetermined cause, the overall waveforms still compared very well.
\end{abstract}

\section{Nomenclature}

$\begin{array}{ll}\boldsymbol{B} & =\text { magnetic flux density } \\ \boldsymbol{H} & =\text { magnetic field } \\ \phi & =\text { angle of polarization rotation } \\ V & =\text { Verdet constant } \\ \mu_{0} & =\text { permeability } \\ \mu_{0} V & =\text { combined permeability Verdet constant } \\ \mathrm{I} & =\text { current } \\ \boldsymbol{I} & =\text { fiber length } \\ N & =\text { number of fiber loops } \\ k A & =\text { kiloamperes }\end{array}$

\footnotetext{
${ }^{1}$ Aerospace Technologist, Electromagnetics and Sensors Branch, MS 130, Hampton, VA 23681.

${ }^{2}$ Aerospace Technologist, Electromagnetics and Sensors Branch, MS 130, Hampton, VA 23681.

${ }^{3}$ Aerospace Technologist, Electromagnetics and Sensors Branch, MS 130, Hampton, VA 23681.

${ }^{4}$ Subject Matter Expert, MS ESC-25.

${ }^{5}$ Electrical Engineer, MS ESC-25.

${ }^{6}$ MLE Electrical \& Instrumentation Lead, MS NE-E8.
} 


\section{Introduction}

G rowing applications of composite materials in commercial aircraft manufacturing has significantly increased the risk of aircraft damage due to lightning attachment. A risk mitigation strategy involves determining lightning current intensitites and distributions on the aircraft from which damage risks could be inferred. Suitable onboard current sensors can be used to measure current intensities and paths during a strike.

For aircraft lightning current measurement, it is desirable to have a current sensor that measures total lightning current directly (not its time derivative), operates down to (near) DC frequency, conforms to aircraft structure, has large measurement ranges, and is light-weight and safe (non-conductive). These characteristics are difficult to achieve in the same traditional sensors applicable for aircraft installation. Many sensors can only measure the timederivatives of the magnetic field or current, and outputs must be integrated to yield desired parameters. Accuracy is a concern at very low frequencies where most of the lightning energy is concentrated. Examples of traditional sensors include B-Dot sensors, I-Dot sensor and Rogowski coil variants. I-Dot and B-Dot sensors, for measuring the time derivatives of the current (I) and magnetic field (B), were used on the NASA F-106 in the Storm Hazard Program in the 1980's ' ${ }^{1}$. Ferromagnetic-core current transformers are self-integrating and can measure current directly. However, aircraft applications are limited due to the large size, weight, and the tendency to saturate in strong currents or magnetic fields. Solid state current sensors based on Hall effect, giant magneto resistive and anisotropic magnetic resistance are often restricted to low bandwidth (up to a few hundred kilohertz) and must be protected from strong fields and currents. A shunt resistor can faithfully provide lightning waveforms, but it requires aircraft structure modifications to provide isolation between the terminals. These sensors typically require fiber optic converters to protect from hazards to personnel and instrumentation inside the aircraft. I-Dot sensors, Rogowski coils, ferrite current transformers and shunt resistors can measure the total current, while others can only measure local current or magnetic field. It is clear that each traditional sensor can satisfy only a few of the desirables previously listed.

This paper explores optical current sensors for aircraft lightning measurement, specifically sensors based on optical fiber. Optical current sensors have been under development for decades. They are beginning to be commercialized, mostly to the power generation and distribution industries. The sensors typically rely on Faraday rotation in which the light's polarization plane rotates when the medium is exposed to a magnetic field. The amount of rotation depends on the medium, the wavelength, and is proportional to the interaction length and the intensity of the magnetic field component in the direction of light propagation.

There are two main groups of optical sensing elements: crystal/bulk-glass based and fiber based. Crystal/bulkglass based sensors can choose from an extensive list of available materials with wide ranges of optical properties. They can have high bandwidth, small size and be immune to vibration. They generally measure only local current or magnetic fields. This type of sensor has been considered for lightning sensing on windmill structures ${ }^{3}$. They are rigid and are difficult to make into closed, conformal forms for measuring total structure currents.

The sensor discussed in this paper is optical fiber based. This sensor type is highly flexible and can measure electric current traveling through large structures. By forming closed loop(s) around aircraft structures, the enclosed current can be measured. Fig. 1 illustrates fiber loops measuring total current flowing through structures of interest. Comparing amplitudes and timings at different locations, current flow paths may be determined. In contrast, dots in Fig. 1 represent possible locations where traditional field sensors, such as B-Dot, could be used. They are primarily for sampling local B-fields. An inverse problem must be modeled and solved for the specific aircraft to approximate current amplitudes ${ }^{1,2}$.

Key advantages of a fiber optic current sensor over traditional sensors include the abilities to conform to large, complex structure geometries. It is self-integrating, thus the output is directly related to the total current. The sensor is also small, light weight, safe from interference, and free of hysteresis and saturation. The sensing fiber is also safe from lightning hazards and can be routed directly into an the aircraft fuselage. The sensor is highly suitable for aircraft applications such as in-flight lightning parameters characterization and can enable inferred damage assessments after a lightning strike. In addition, it can also be used on internal structures, including fuel tanks, for system health monitoring or measuring lightning transfer function for certification purposes. Other uses such as on windmill structures are also possible.

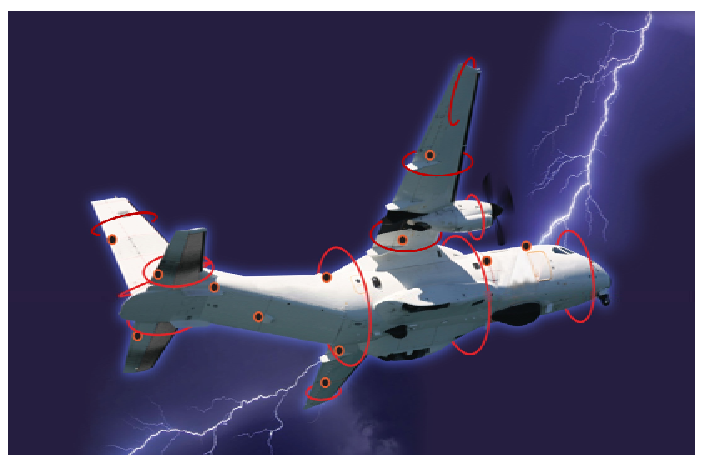

Figure 1. Fiber-optic Sensors Installations. 
The material choice for optical fiber is much more limited than for bulk-glass/crystals - most commonly available fiber materials are based on silica. The Faraday effect in silica is weak, which makes it ideal for large currents in lightning. However, temperature and bend/vibration sensitivities could be of concern depending on designs. The fiber is also fragile and needs suitable protection.

In the remainder of the paper, basic sensor operation, design, and bandwidth are discussed. Laboratory test results are reported. Finally, results from a field evaluation measuring rocket-triggered lightning current are presented. The Faraday rotation fiber optic current sensor is simply referred to as Faraday sensor in this paper.

\section{Generalized Lightning Environment}

Generalized aircraft lightning environments are summarized in SAE/ARP 5412A ${ }^{4}$. The peak lightning current for direct effect testing can be as high as $200 \mathrm{kA}$ or greater (component $\mathrm{A}$ ). The long duration continuing current can be about $200 \mathrm{~A}-800 \mathrm{~A}$ (component C). Thus the measurement range needs to be $60 \mathrm{~dB}(200 \mathrm{~A}-200 \mathrm{kA})$ or greater. Most of the lightning energy is limited to frequency spectrum far below $1 \mathrm{MHz}$. This is an important consideration in selecting the fiber optic sensor length and bandwidth.

\section{Fiber Optic Current Sensor Solution}

For the Faraday rotation effect, light polarization in an optical medium rotates when the medium is exposed to a magnetic field in the direction of light propagation. The effect in optical fiber is illustrated in Fig. 2. $\phi$ (in radians) is the change to polarization $\boldsymbol{E}$ as a function of the magnetic field, the medium's Verdet constant $V$ and length $\boldsymbol{I}$. The polarization plane rotation is given as ${ }^{5}$ :

$$
\phi=V \int \boldsymbol{B} \cdot d \boldsymbol{l}=\mu_{0} V \int \boldsymbol{H} \cdot d \boldsymbol{l},
$$

where $\mu_{0}$ is the free-space permeability; $V$ is the Verdet constant in radians/(meter-Tesla); $\mu_{0} V$ is the combined permeability Verdet constant (radians/ampere); $\boldsymbol{B}$ is magnetic flux density in Tesla (T); length $\boldsymbol{I}$ (in meters) is the light and magnetic field interaction path length; and $\boldsymbol{H}$ is the magnetic field (amperes/meter). For a fiber forming $N$ closed loops around a conductor carrying current $I$ (ampere), applying Ampere’s law yields:

$$
\begin{aligned}
\phi & =\mu_{0} V \oint \boldsymbol{H} \cdot d \boldsymbol{l}, \\
& =\mu_{0} V N I .
\end{aligned}
$$

Faraday rotation is a form of circular birefringence, where circularly polarized light propagates through the medium at different speeds for the left-handed and the right-handed polarizations. Since linear polarization can be decomposed into the two circularly polarized components, different propagation speeds in a magnetized medium result in the rotation of the plane of polarization. Circular

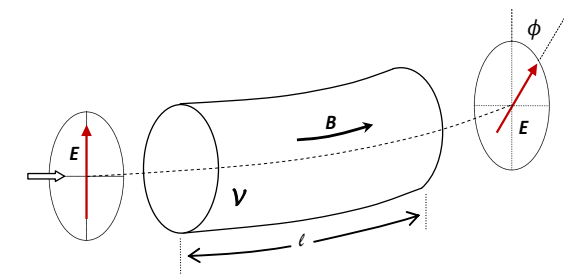

Figure 2. Faraday rotation in optical fiber. birefringence is desirable in measuring current.

A twisted single-mode fiber is used in this setup. Twisting the fiber is an approach to introduce circular birefringence through torsion strain. Twisting helps maintain the linear state of polarization (SOP) otherwise destroyed by accumulation of linear birefringence. Linear birefringence, where light beams in the two orthogonal linear polarizations propagate at different speeds, are caused by intrinsic stresses (such as core deformation) and extrinsic stresses (fiber bending, compression, etc.). Large accumulation of linear birefringence can interact with and destroy the effects of circular birefringence and the polarization state. Evolution of light polarization in twisted single-mode fiber has been studied extensively. Further discussions can be found in Ref. 6-10.

\section{A. Basic System Setup}

A prototype sensor system based on a polarimetric scheme was developed for this study. In a typical basic polarimetric scheme, linearly polarized light is transmitted though the sensing fiber, and the detection of the polarization rotation is performed with a polarizer and a detector. This approach can measure DC current while having the sufficient bandwidth to include the high energy portion of the lightning spectrum. The setup presented in this paper extends the basic scheme to include a Faraday mirror. In addition, a dual-detectors setup measures the two orthogonal polarizations for increased sensitivity. 
The setup is illustrated in Fig. 3. A linearly polarized light from a super-luminescence diode (SLD) laser is generated at locations labeled 1, 2. Half of the power is transmitted through the nonpolarizing beam splitter (NBS) at 3 to the sensing fiber at 4 . The sensing fiber forms closed loops around the current carrying conductor at 5 . A Faraday mirror at 6 rotates the reflected light polarization by $90^{\circ}$ relative to the incident light. This cancels out fiber bend/stress induced effects, as effects to one polarization in the forward direction are similarly imposed onto the orthogonal polarization on the return trip. The effect makes the sensor less sensitive to bending. The reflected

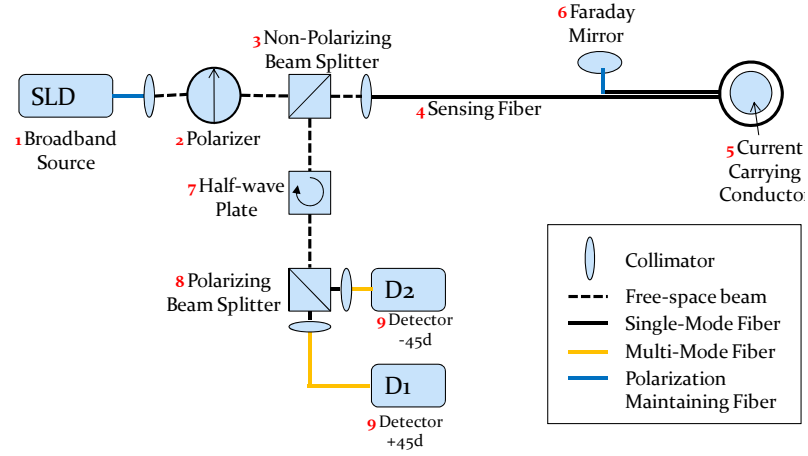

Figure 3. Faraday sensor setup.

light traces back through the fiber to 3 , at which half of the power is reflected through the half-wave plate (HWP) at 7 toward the polarizing beam splitter (PBS) at 8. In short, the NBS is acting as an inefficient circulator where only $25 \%$ of the original power reaches the PBS. Exiting the PBS at 8, light power in the two orthogonal polarizations are measured by two photo-detectors D1 and D2 at 9. The HWP helps rotate and align the initial polarization incident on the PBS at zero current. Ideally, at zero current the incident polarization should be at $45^{\circ}$ relative to the PBS's two orthogonal principle polarization axes, so that beam power is divided equally between the two optical detectors at 9. This setup is referred to as a reflective scheme, since a mirror is used. In this reflective scheme with a Faraday mirror, the non-reciprocal Faraday rotation is doubled due to the round-trip around the conductor; while stress induced polarimetric effects are cancelled. A balance detector, with two built-in matched detectors, is used in place of two separate detectors. This helps subtract common-mode noise between the two detectors and significantly improves noise performance.

When the sensing fiber is exposed to a current induced magnetic field, light traveling through it experiences polarization shifts. These shifts cause power changes detected by the optical detectors. The output voltage waveforms from the detectors are used in calculating the current on the conductor.

Fig. 4 shows key components of the setup including the wideband SLD source, the optical table, and a Faraday mirror. The SLD laser is a $850 \mathrm{~nm}$ broadband source with a polarizationmaintaining output fiber. The spectrum is Gaussian-like with approximately $50 \mathrm{~nm}(-3 \mathrm{~dB})$ bandwidth. The 50-meter long sensing fiber is made from a standard telecom single-mode fiber (HI 780) and is twisted at 20 twists per meter. The output beams from the optical table are routed to the two detectors (in the balance detector unit) via two multi-mode fibers.

The responses at the two detectors should ideally be ${ }^{5}$ [a],[b] $=0.5 *\left[1 \pm \sin \left(4 \mu_{0} V N I\right)\right]$ as labelled in Fig. 5 . It is important to note that the total Faraday rotation in this reflective scheme is twice that for the single-pass scheme (no mirror). A typical operating range is where the response increases monotonically with current, such as between $-150 \mathrm{kA}$ to +150 kA. While the output from either detector is suitable for measurement, performing "difference-over-sum" math operation $[\mathrm{c}]=\{[\mathrm{a}]-[\mathrm{b}]\} /\{[\mathrm{a}]+[\mathrm{b}]\}$ and trigonometry simplification would result in a response that is twice as sensitive and has a zero crossing at zero current. Thus,

$$
\begin{aligned}
& {[\mathrm{c}]=\sin \left(4 \mu_{0} V N I\right), \text { or }} \\
& N I=\frac{1}{4 \mu_{0} V} \sin ^{-1}[\mathrm{c}],
\end{aligned}
$$

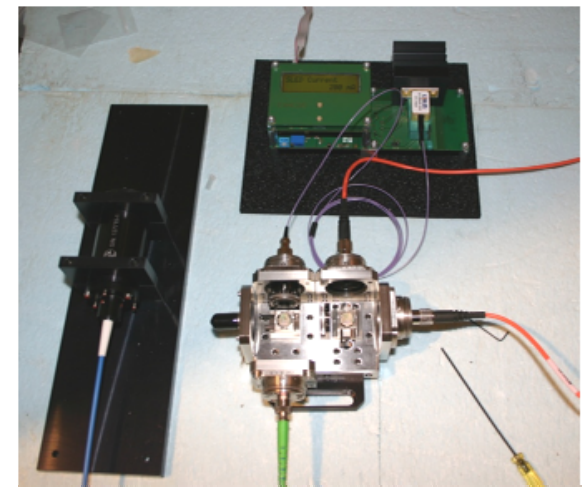

Figure 4. Key components: Faraday mirror (left), laser (top), and optical

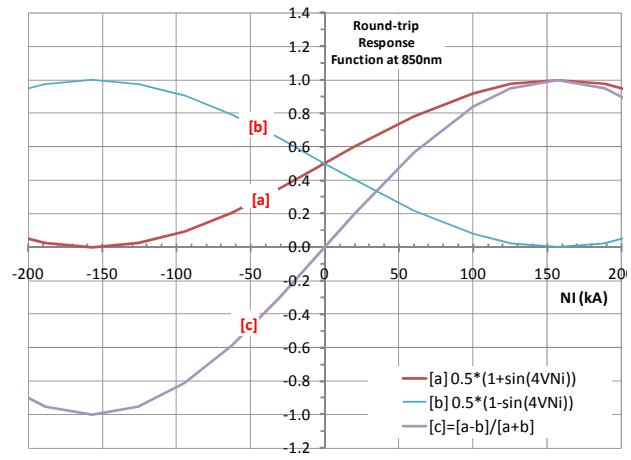

Figure 5. Reflective scheme response functions at $850 \mathrm{~nm}$. 
where $N I$ is the number of loops $N$ times the current $I$, and $\mu_{0} V=2.5 \times 10^{-6} \mathrm{rad} / \mathrm{A}$ at $850 \mathrm{~nm}{ }^{5}$. The difference operation is actually performed with the balance detector to yield only one voltage waveform output.

Operating at 850nm center wavelength, the sensor can measure from approximately $140 \mathrm{kA}$ down to about 200 A (limited by the SLD laser noise), a $57 \mathrm{~dB}$ range. With low pass filtering and data smoothing, a $60 \mathrm{~dB}$ range can be achieved. This is an improvement over an earlier setup ${ }^{11}$ with a $34 \mathrm{~dB}$ range.

\section{B. Laboratory Testing}

A low-current laboratory lightning waveform generator, capable of $2 \mathrm{kA}$, was used in the testing and characterizing the sensor. Two approaches were used to produce the Faraday effect at levels associate with measuring natural lightning current. In one setup, the $50 \mathrm{~m}$ long sensing fiber formed a 94-turn coil around a conductor, amplifying the Faraday rotation by the same factor. This setup produced a weak and nearly uniform magnetic field over the entire $50 \mathrm{~m}$ fiber. In the second setup, a 100-turn solenoid subjected a short fiber segment to strong magnetic field levels similar to that produced by natural lightning. Fig. 6 shows the two setups. Effects associate with currents up to approximately $140 \mathrm{kA}$ could be simulated. A Pearson's current transformer and a

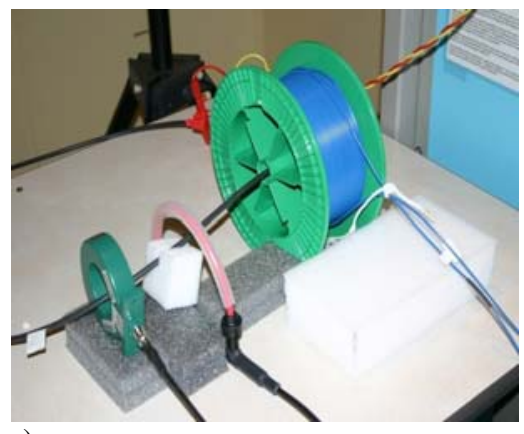

a)

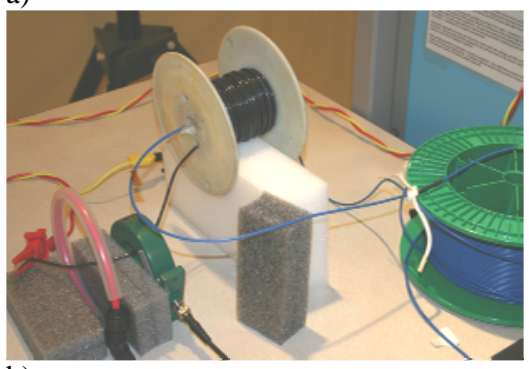

b)

Figure 6: Using multiple fiber loops or a coil winding to simulate effects due to higher current.

Rogowski coil were used as reference, measuring current on a single wire. Their data were compared to the Faraday sensor by first numerically scaling up by the number of fiber loops on the spool (94) or the number turns on the wire coil (100), depending on the setup.

A series of measurements were made with current transients having different amplitudes. For each transient, the peak values of the waveforms from the sensors were recorded. Eq. 3 was then applied to produce the Faraday sensor current data, which were plotted against the Pearson's current transformer data as shown in Fig. $7 . \quad$ The Rogowski data were similar to the Pearson's probe data and used as an independent check. Though not shown here, the resulting characteristic

curves for the two setups were almost identical, indicating suitability for both weakly distributed current as well as strong concentrated current, or combinations of both. Thus, the same sensor could be used on large structures such as a fuselage that may have lower level distributed current, or on small structures like tails and wing tips with more concentrated current. The measured results would be identical, demonstrating the sensor's versatility.

In an ideal setup, the plot of Faraday sensor current versus a reference sensor current would fall on a straight line labeled as "ideal" in Fig. 7. In practice, the data comparison followed the blue curve labed "uncorrected" in Fig. 7. The lower slope in the linear region indicated a reduced sensitivity of about $92 \%$ of ideal. Beyond +/-50 kA the curve started to become nonlinear. This deviation was caused by weak stray optical beams from non-ideal optical components, causing optical biases at the detectors.

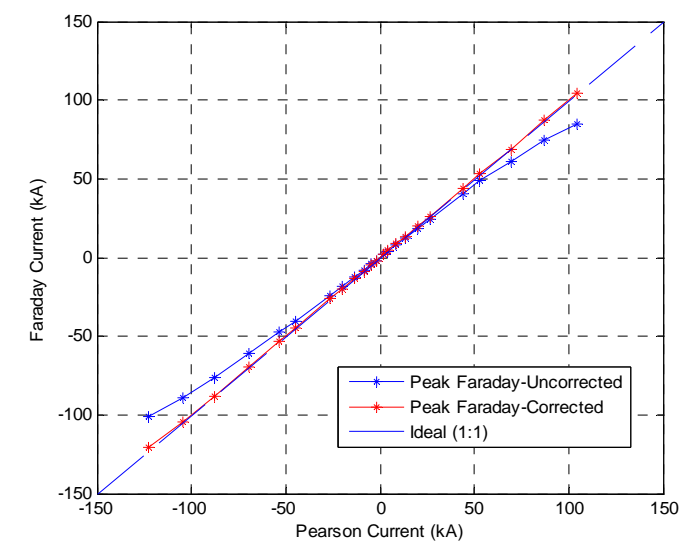

Figure 7. Faraday sensor's uncorrected and corrected (linearized) response curves.

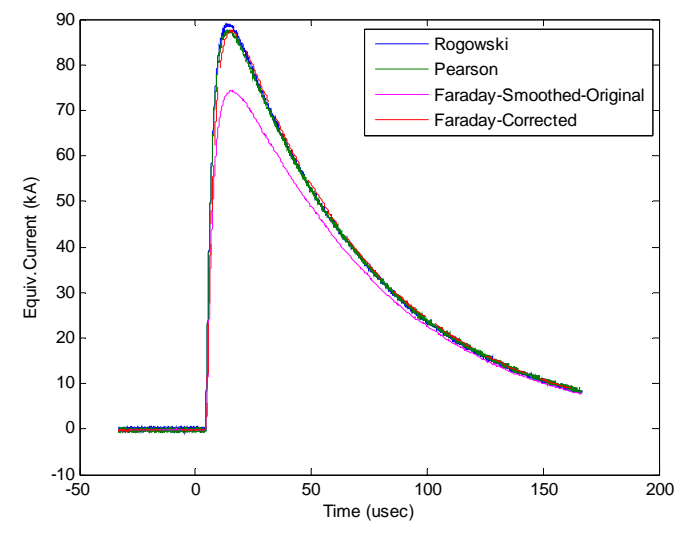

Figure 8. Faraday sensor comparison against reference sensors for a $90 \mathrm{kA}$ current waveform. 
To correct both the reduced sensitivity and the non-linear response, a simple fifth-order spine-fit "correction" function was developed from Fig. 7 to map the Faraday sensor response to the "ideal" curve. This function was then applied to the subsequent measured waveforms. Fig. 7 illustrates the corrected response curve aligns well with the ideal curve. Fig. 8 illustrates the "uncorrected" and "corrected" Faraday sensor data against the reference sensors for a $90 \mathrm{kA}$ peak current waveform. Good comparison was achieved after the correction.

\section{Sensor Bandwidth}

Bandwidth of a sensor system is limited by the lowest bandwidth of its components. For the fiber sensor component, it is limited by the light transit time in the interaction length of the fiber. This bandwidth limitation is to ensure the total transit time is much faster than the signal change rate. The fiber interaction length in the bandwidth consideration includes the roundtrip length around the conductor and includes the length to and from the Faraday sensor. The 3-dB sensor bandwidth $B W$

Table 1. Structure Dimensions vs. Sensor Bandwidths

\begin{tabular}{|c|c|c|c|}
\hline $\begin{array}{c}\text { 3-dB } \\
\text { Bandwidth } \\
\text { (MHz) }\end{array}$ & $\begin{array}{c}\text { Max. Fiber } \\
\text { Length (m) }\end{array}$ & $\begin{array}{c}\text { Max. Thin } \\
\text { Structure } \\
\text { Dimension (m) }\end{array}$ & $\begin{array}{c}\text { Max. Round } \\
\text { Structure } \\
\text { Diameter (m) }\end{array}$ \\
\hline 1 & 44 & 22 & 14 \\
\hline 2 & 22 & 11 & 7 \\
\hline 4 & 11 & 5.5 & 3.5 \\
\hline 10 & 4.4 & 2.2 & 1.4 \\
\hline 20 & 2.2 & 1.1 & 0.7 \\
\hline
\end{tabular}

is ${ }^{5}: B W \approx \frac{0.44}{t} \approx 0.44 c / n L$, where $t$ is transit time,

$c$ is the speed of light in free space, $n$ is the index of refraction in fiber material $(n=1.5)$, and $L$ is the interaction length (double of fiber length for reflective scheme).

Table 1 computes the maximum fiber length and structure dimensions for different bandwidths. Aircraft thin structures may include wings and tail surfaces, while round structures may include fuselage, engine, etc. For reference, fuselage outside diameters for various aircraft (averaging the width and height) include: Airbus A380: 7.8 $\mathrm{m}$; Boeing 767: $5.3 \mathrm{~m}$; Boeing 737: $3.8 \mathrm{~m}$. Assuming most of the damaging lightning energy is far below 1-2 $\mathrm{MHz}$, the table shows there is sufficient sensor bandwidth even for the fuselage of the largest passenger aircraft, the Airbus A380.

\section{Triggered Lightning Sensor Evaluation}

The sensor system was evaluated measuring rockettriggered lightning at the International Center for Lightning Research and Testing (ICLRT) facility in Camp Blanding, FL ${ }^{12}$ over the summer of 2011. In the setup in the background of Fig. 9, triggered lightning flashes would attach to the wire cage, and the currents would travel to the ground via a shunt resistor and a down-conductor. A part of the $50 \mathrm{~m}$ sensing fiber formed two closed loops around the conductor as shown in Fig. 10. The remaining fiber segments at the two ends were co-routed radially from the site to minimize loop area and any possible effects due to ground current. The two ends were then connected to the Faraday mirror and the optical box located 12 $\mathrm{m}$ away. The fiber was protected inside rain gutters and a garden hose from wild animals and the weather. In addition, the Faraday mirror was buried in the ground to minimize

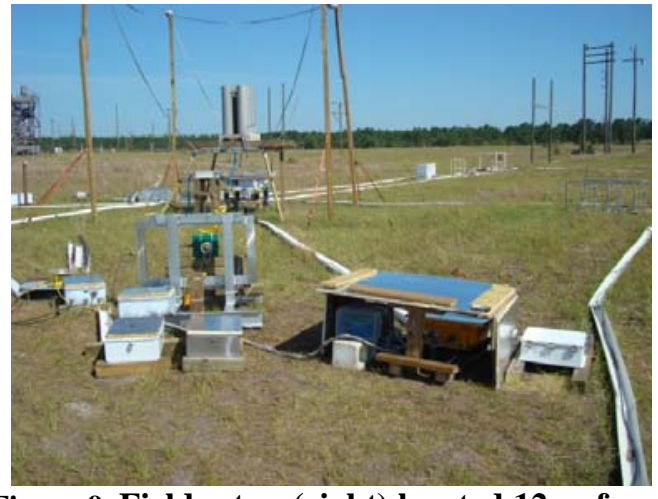

Figure 9. Field setup (right) located $12 \mathrm{~m}$ from the rocket launch pad. concerns about temperature sensitivity. With the two fiber loops used, the system was twice as sensitive as a single loop, while the maximum amplitude range was halved. Thus the system would be able to measure $100 \mathrm{~A}$ to $70 \mathrm{kA}$.

For bandwidth consideration, it is noted that the fiber interaction length was only from the start of fiber loops to the Faraday mirror and back, not the entire $50 \mathrm{~m}$. Thus, for the approximately $20 \mathrm{~m}$ fiber length (two times the loops length and the distance to the Faraday mirror), the 3-dB measurement bandwidth was about $2 \mathrm{MHz}$.

A 14-bit data acquisition system was set to capture at 100 mega samples per second in a $30 \mathrm{~ms}$ time window. Multiple strokes in a flash could be recorded with the system. The system had a $50 \mathrm{ohm}$ input impedance through a terminating resistor. A current shunt (T\&M Model R-7000-10) located directly above the two fiber loops (box) served as reference. 
During May - August 2011, nine lightning flashes were captured - each typically contained more than one stroke. Fig. 11 shows the results for two of the five strokes in the first flash captured. The results compared very well against the reference shunt measurement. As can be seen, current down to $0.11 \mathrm{kA}$ could be observed at $t=1.89 \mathrm{~ms}$ in Fig. 11.b. The good result comparison demonstrated the accuracy and feasibility in a real lightning environment. It is noted that the Faraday Sensor data were smoothed using a 20-points window for noise reduction. In comparison, 100 thousand data points were record and shown in each 1 ms window.

Subsequent measurements, however, show amplitude deviations from the shunt resistor that gradually became worse with time. In the second flash, Faraday sensor data were about $10 \%$ higher than reference shunt resistor data. By the end of the

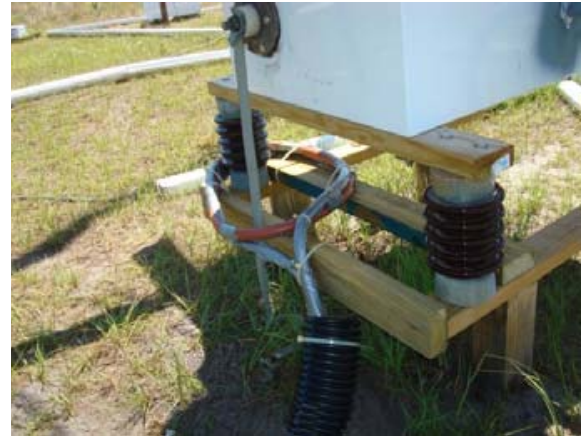

Figure 10. Two fiber loops (inside a garden hose) measuring current exiting the shunt resistor (box). summer the difference was as much as $30 \%$. The cause is being investigated, though a post measurement laboratory evaluation did not show a significant change in the Faraday sensor system performance. Only 2-3\% sensitivity reduction was observed, which was caused by a slight optical beam mis-alignment or defocusing. In spite of the amplitude differences, the waveform shapes still compared very well when the amplitudes were (arbitrarily) scaled to match. Fig. 12 shows the comparison of data from the second flash recorded 2 weeks later. The Faraday sensor data were scaled down by $10 \%$ in this comparison.

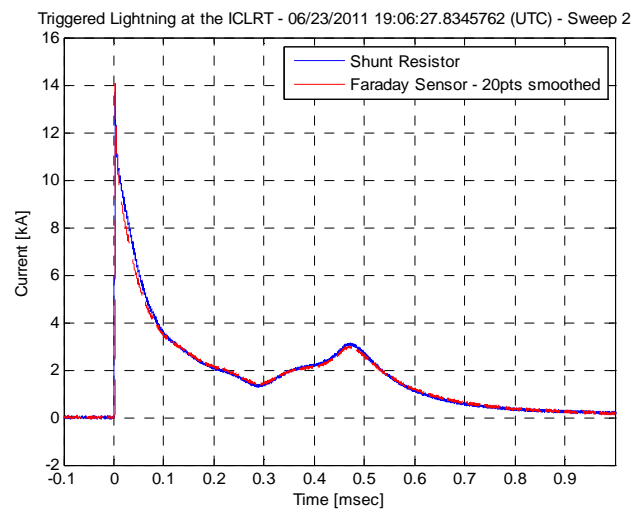

a)

Figure 11. Results comparison for two strokes in the first lightning flash.

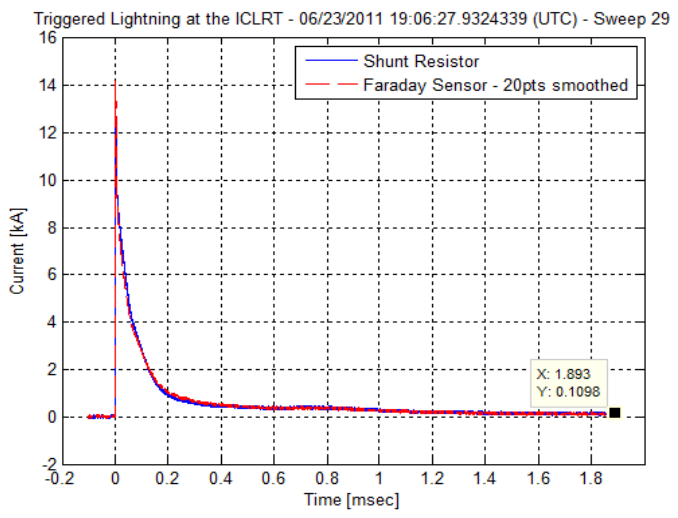

b)

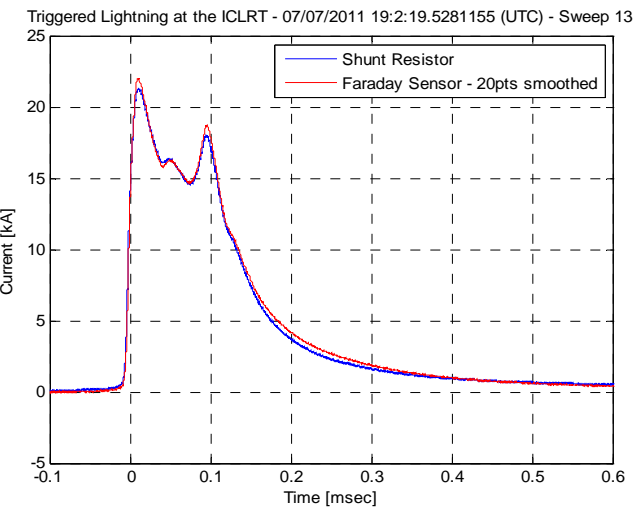

a)

Figure 12. Waveform comparison in subsequent measurements. Faraday sensor data scaled to $90 \%$.

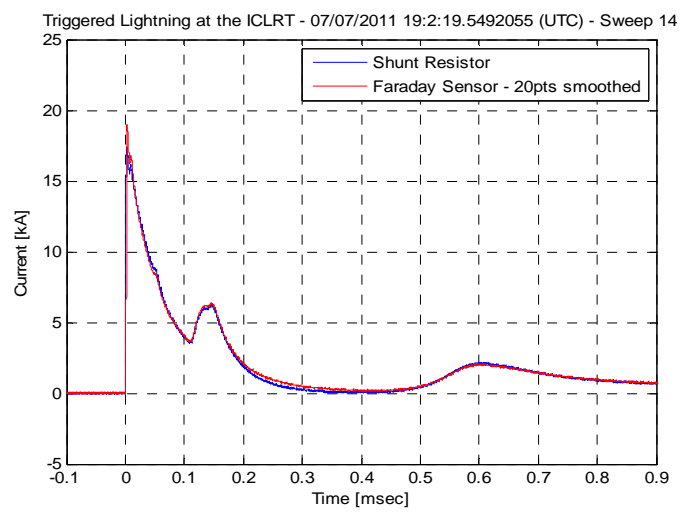

b) 


\section{E. Future Work}

Fiber breakage and temperature sensitivity of twisted fiber are a concern for long term use. As a result, future systems will include spun fibers that are gaining acceptance in the power industry. Spun fibers are twisted during manufacturing, and are typically made from polarizating-maintaining fibers rather than with single-mode fiber as with twisted fiber. Spun fibers have different characteristics than twisted fiber, and are generally more immune to breakage and bend sensitivity. In addition, systems operating different wavelengths will be constructed for different amplitude ranges. A sensor system based on $1550 \mathrm{~nm}$ wavelength has been built that can measure current up to 400 kA due to the reduced sensitivity from the lower Verdet constant. The system is being field tested at the ICLRT during summer 2012. A different approach may be considered in future setups to improve range and to minimize post measurement linearization as required in the present polarimetric scheme.

\section{Conclusion}

This paper describes a unique current sensor system based on Faraday effects in optical fiber for measuring lightning current. The sensor system has numerous advantages over traditional sensors and represents a significant leap in the ability to measure total current on arbitrarily shaped structures. Results demonstrate good accuracy can be achieved in the laboratory and in a realistic lightning environment. Future improvements in range, reliability and cost of the system could potentially lead to flight tests and eventual aircraft implementation.

\section{References}

${ }^{1}$ Pitts, F. L., Fisher, B. D., Mazur, V., and Perala, R. A., “Aircraft Jolts from Lightning Bolts,” IEEE Spectrum, July 1988.

${ }^{2}$ Alestra, S., Revel, I., Srithammavanh, V., Bardet, M., Zwemmer, R., Brown, D., Marchand, N., Ramos, J., Stelmashuk, V. "Developing an In-Flight Lightning Strike Damage AssessmentSystem,” ICOLSE, 2007.

${ }^{3}$ Krämer, S. G. M., and León, F. P., "Fiber-Optic Current Sensors for Lightning Detection in Wind Turbines,” OSA/OFS, 2006.

${ }^{4}$ SAE/ARP5412A “Aircraft Lightning Environment and Related Test Waveforms,” 2005.

${ }^{5}$ Lopex-Higuera, J. M., Editor. Handbook of Optical Fibre Sensing Technology, 2002; Sections 27.2 - 27.4.

${ }^{6}$ Smith, A. M., "Polarization and Magneto-optic Properties of Single-Mode Optical Fiber,” Applied Optic, Jan. 1978.

${ }^{7}$ Ulrich, R., and Simon, A., "Polarization Optics of Twisted Single-Mode Fibers," Applied Optics, Vol. 18, Issue 13, pp. 2241-2251 (1979).

${ }^{8}$ Rashleight, S. C., “Origins and Control of Polarization Effects in Single-Mode Fibers”, Journal of Lightwave Technology, Vol. LT-1, No. 2, 1983.

${ }^{9}$ White, A. D., McHale G. B., and Goerz, D. A., "Advances in Optical Fiber-Based Faraday Rotation Diagnostics", 17th IEEE Intl. Pulsed Power Conf., 2009.

${ }^{10}$ Day, G. W., and Rose, A. H., "Faraday Effect Sensors: The State of the Art,” Proc. SPIE, 1988, pp. 138-150.

${ }^{11}$ Nguyen, T. X., and Szatkowski, G. N., "Fiber Optic Sensor for Aircraft Lightning Current Measurement”, ICOLSE, 2011.

${ }^{12}$ Rakov, V. A., "A review of Triggered-Lightning Experiments," 30th International Conference on Lightning Protection, Cagliari, Italy, September 13-17, 2010. 